\title{
ESTUDO BIOMECÂNICO DA FIXAÇÃO PEDICULAR CURTA NA FRATURA-EXPLOSÃO TORACOLOMBAR
}

\author{
BIOMECHANICAL EVALUATION OF SHORT-SEGMENT FIXATION FOR THORACOLUMBAR \\ BURST-FRACTURES
ESTUDIO BIOMECÁNICO DE FIJACIÓN PEDICULAR CORTA EN LA FRACTURAEXPLOSIÓN TORACOLUMBAR

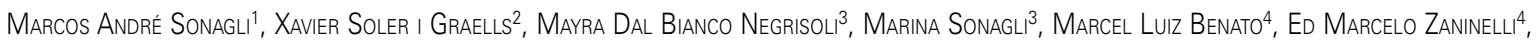
Luciane Yumi Suzuki de Olivelra ${ }^{5}$, Luiz Antonio Munhoz da Cunha ${ }^{6}$

\begin{abstract}
RESUMO
Objetivo: Comparar a rigidez biomecânica entre a coluna toracolombar intacta, a coluna com fratura explosão e a coluna com fratura explosão associada à fixação pedicular curta em suínos. Métodos: 30 amostras de coluna toracolombar (T11-L3) de suínos foram divididas em três grupos com 10 amostras cada. O Grupo 1 representava a coluna intacta, o Grupo 2 representava a coluna com fratura explosão e o Grupo 3 a fratura explosão associada à fixação pedicular curta. Foi realizado o corte ósseo em "V" do terço médio do corpo vertebral comprometendo a coluna anterior e média de L1 para simular a fratura explosão. No Grupo 3 foi realizada a fixação pedicular com Pinos de Schanz. Os grupos foram submetidos ao teste biomecânico em compressão axial controlada. Os parâmetros de carga (N) e deslocamento $(\mathrm{mm})$ eram gerados em um gráfico instantâneo e a rigidez $(\mathrm{N} / \mathrm{mm})$ foi determinada. O teste era interrompido quando ocorria uma queda súbita na curva no gráfico indicando falência da amostra. Resultados: A rigidez das colunas fraturadas foi $53 \%$ menor do que a rigidez das colunas intactas, sendo essa diferença estatisticamente significativa $(p<0,05)$. A fixação pedicular curta apresentou uma rigidez 50\% maior do que a coluna fraturada. Esse aumento foi estatisticamente significativo $(p<0,05)$. A rigidez da fixação pedicular curta foi 30\% menor do que a rigidez das colunas intactas. Essas diferenças foram estatisticamente significativas $(p<0,05)$. Conclusão: A fixação pedicular curta não é suficiente para restabelecer a rigidez da coluna intacta nos testes biomecânicos in vitro de compressão axial pura em modelos de fratura toracolombar de suínos.
\end{abstract}

Descritores: Fraturas da coluna vertebral; Biomecânica; Suíno.

\begin{abstract}
Objective: Compare the biomechanical stiffness between the intact spine, the spine with burst fracture and the short-segment pedicle fixation on porcine thoracolumbar burst fracture. Methods: 30 samples of thoracolumbar spine (T11-L3) of porcine were divided into three groups with 10 samples each. Group 1 represented the intact spine, Group 2 the spine with burst fracture and Group 3 the burst fracture associated with short-segment pedicle fixation. The burst fracture injury was created with a "V" shape cut of the third middle of the vertebral body compromising the L1 anterior and medial columns simulating the burst fracture. Group 3 was stabilized with Schanz pedicle screws. The groups were subjected to biomechanical testing in a controlled axial compression. The parameters of load ( $\mathrm{N})$ and displacement (mm) were generated in a graphic snapshot and stiffness $(\mathrm{N} / \mathrm{mm})$ was determined. The test was stopped when there was a sudden drop in the curve on the chart indicating failure of the sample. Results: The stiffness of the fractured spines was 53\% lower than the stiffness of the intact spine and this difference was statistically significant $(p<0.05)$. The stiffness of the short-segment pedicle fixation was $50 \%$ higher than the fractured spine. This difference was statistically significant $(p<0.05)$. The stiffness of the short-segment pedicle fixation was $30 \%$ lower than the intact spine. These differences were statistically significant $(p<0.05)$. Conclusion: The short-segment pedicle fixation does not provide sufficient stability to restore the stiffness of the intact spine during pure axial load compression biomechanical testing.
\end{abstract}

Keywords: Spinal fractures; Biomechanics; Porcine.

\section{RESUMEN}

Objetivo: Comparar la rigidez biomecánica entre la columna toracolumbar intacta, la columna con fractura-explosión y la columna con fractura-explosión asociada a la fijación pedicular corta en cerdos. Métodos: 30 muestras de columna toracolumbar (T11-L3) de cerdos fueron divididas en tres grupos con 10 muestras cada una. El Grupo 1 representaba la columna intacta, el Grupo 2 representaba la columna con fractura-explosión y el Grupo 3 la fractura-explosión asociada a la fijación pedicular corta. Fue realizado el corte óseo en "V" del 1/3 medio del cuerpo vertebral, comprometiendo la columna anterior y media de L1, para simular la fractura-explosión. En el Grupo 3 fue realizada la fijación pedicular con pernos de Schanz. Los Grupos fueron sometidos al test biomecánico en compresión axial controlada. Los parámetros de carga $(N)$ y desplazamiento $(\mathrm{mm})$ eran generados en un gráfico instantáneo y la rigidez $(\mathrm{N} / \mathrm{mm})$ fue determinada. El

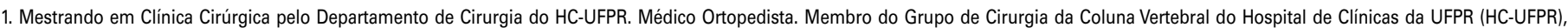
Curitiba, PR, Brasil.

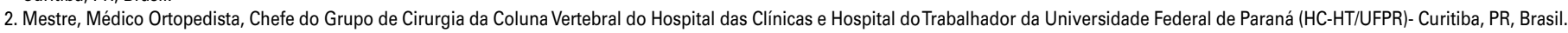
3. Acadêmica de Graduação da Faculdade de Medicina da Universidade Federal de Paraná (UFPR)- Curitiba,PR, Brasil.

4. Médico Ortopedista, Membro do Grupo de Cirurgia da Coluna Vertebral do Hospital das Clínicas e Hospital doTrabalhador da Universidade Federal de Paraná (HC-HT/UFPR)- Curitiba, PR, Brasil.

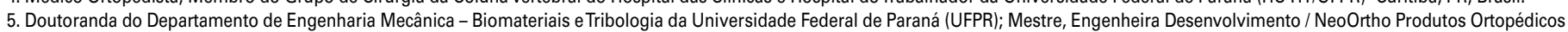
SA - Curitiba, PR, Brasil.

6. Mestre, Doutor, Médico Ortopedista.Chefe do Serviço de Ortopedia eTraumatologia do Departamento de Cirurgia do Hospital de Clínicas da UFPR, Curitiba, Brasil.

Trabalho realizado no Grupo de Cirurgia da Coluna Vertebral do Hospital das Clínicas e Hospital doTrabalhador da Universidade Federal de Paraná - (HC-HT/UFPR).

Correspondência: Rua Monsenhor Ivo Zanlorenzi, 1668 ap.1501. Curitiba - Paraná - Brasil. CEP: 81210-000. E-mail: msonagli@yahoo.com 
test era interrumpido cuando ocurría una caída súbita en la curva en el gráfico señalando la falla de la muestra. Resultados: La rigidez de las columnas fracturadas fue 53\% menor que la rigidez de las columnas intactas, siendo esta diferencia estadísticamente significativa ( $p<$ 0,05). La fijación pedicular corta presentó una rigidez $50 \%$ mayor que la columna fracturada. Este aumento fue estadísticamente significativo $(p<0,05)$. La rigidez de la fijación pedicular corta fue $30 \%$ menor que la rigidez de las columnas intactas. Estas diferencias fueron estadísticamente significativas $(p<0,05)$. Conclusión: La fijación pedicular corta no es suficiente para restablecer la rigidez de la columna intacta en las pruebas biomecánicas in vitro de compresión axial pura en modelos de fractura toracolumbar de cerdos.

Descriptores: Fracturas de la columna vertebral; Biomecánica; Porcinos.

\section{INTRODUÇÃO}

As fraturas da coluna toracolombar representam aproximadamente 70 a $80 \%$ das fraturas da coluna vertebral, 35\% na região torácica e $40 \%$ na região lombar ${ }^{1}$. De todos os subtipos de fratura da coluna vertebral, a fratura do tipo explosão é a mais comum, com cerca de $60 \%$ dos casos de tratamento cirúrgico².

A fratura explosão toracolombar ocorre quando o corpo vertebral é sujeito a uma força axial significativa provocando a falha por compressão das colunas anterior e média da coluna vertebral ${ }^{3-5}$. A maioria das fraturas-explosão envolve a junção toracolombar, que é particularmente susceptível a este tipo de lesão por causa de sua anatomia transicional entre a coluna torácica cifótica e menos móvel e a coluna lombar lordótica e mais móvel. Esta transição gera um movimento de força em dobradiça (braço de alavanca) sobre a região anterior do corpo vertebral, gerando instabilidade e deformidade cifótica progressiva da coluna vertebral ${ }^{6}$.

Uma das formas de tratamento deste tipo de lesão é o tratamento cirúrgico que tem como objetivos: proporcionar a correção da deformidade cifótica (ângulo de correção), recuperar a estabilidade da coluna vertebral, realizar a descompressão indireta do canal medular, permitir a reabilitação e a mobilização precoce do paciente ${ }^{7,8}$.

Para realizar o tratamento cirúrgico desta lesão, diversas abordagens e técnicas de fixação têm sido propostas, entre elas: abordagem posterior, abordagem anterior e abordagem combinada (anterior + posterior) ${ }^{2}$.

Pela abordagem posterior são realizadas as técnicas de fixação pediculares curta e longa. A fixação pedicular curta inclui a fixação de uma vértebra acima e uma vértebra abaixo da fratura ${ }^{9}$. A fixação pedicular longa inclui a fixação de duas ou mais vértebras acima e duas ou mais vértebras abaixo da fratura. Cada técnica utilizada apresenta vantagens e desvantagens quando comparadas entre si. Alguns estudos demonstram que a fixação pedicular longa proporciona uma estabilidade biomecânica superior, porém à custa de perda da mobilidade toracolombar, já que é realizada a fixação de mais segmentos ${ }^{10}$. Embora a fixação pedicular curta apresente algumas vantagens decorrentes da instrumentação de menos níveis, existem elevados índices de perda de redução e falha do material de implante devido à falta de suporte anterior da coluna vertebral $^{11}$. Para melhor compreender os motivos da falência da fixação pedicular curta torna-se necessário avaliar as características biomecânicas deste tipo de instrumentação.

A capacidade de um sistema de instrumentação vertebral de prover estabilidade depende de duas propriedades mensuráveis: capacidade de absorver carga até sua falha (fadiga) e sua rigidez. A rigidez do sistema de instrumentação é responsável pela estabilidade inicial da coluna vertebral logo após a fixação da fratura e é durante este período inicial do pós-operatório (antes da consolidação da fratura) que ocorrem as perdas de redução com a fixação pedicular curta ${ }^{2,11,12}$.

Portanto, o objetivo deste estudo é avaliar a rigidez biomecânica da fixação pedicular curta e compará-la com a rigidez biomecânica da coluna vertebral íntegra e da coluna vertebral com fratura explosão, para uma melhor compreensão dos motivos da falência inicial deste tipo de instrumentação.

\section{MATERIAIS E MÉTODOS}

\section{Análise de cálculo de amostragem}

Após avaliação estatística foi definida o número mínimo de 10 amostras de coluna toracolombar por grupo de estudo (total de 30 amostras), para que fosse necessário verificar diferenças estatísticas entre os grupos.

\section{Definição dos grupos}

Para verificação da hipótese foi realizado a divisão das amostras em três grupos distintos:

Coluna Vertebral Intacta - Grupo Controle;

Coluna Vertebral com fratura explosão;

Coluna vertebral com fratura explosão + Fixação pedicular curta.

\section{Preparação das amostras}

O total de 30 amostras de coluna toracolombar de suíno da mesma raça foi adquirido de um frigorífico local. Estas amostras foram liberadas de acordo com as leis sanitárias locais.

Todas as amostras foram envolvidas em saco plástico duplo e armazenadas em um freezer com temperatura de $-20^{\circ} \mathrm{C}$ (Celsius). Antes da realização do teste biomecânico, as amostras foram descongeladas em temperatura ambiente por 12 horas. Todos os tecidos musculares foram removidos, deixando intactos: as vértebras, os discos e ligamentos espinhais. Para evitar a influência da exposição ao ar (ressecamento) no comportamento biomecânico, todas as amostras foram umidificadas com soro fisiológico $(\mathrm{NaCl}$ $0,9 \%$ ) antes e durante os testes. Após a preparação das amostras conforme descrito acima, cada uma foi aferida quanto às dimensões e peso. Para amostragem adequada, foram selecionados amostras de coluna suínas de dimensões e pesos semelhantes. Após, foram identificadas e numeradas em ordem sequencial conforme o grupo de estudo.

O manuseio e a preparação das amostras desta forma, rotineiramente utilizada nas análises biomecânicas, não alteram as características materiais do osso e das partes moles ${ }^{13}$. Este protocolo foi utilizado conforme preconizado pelos estudos biomecânicos descritos por Lundin et al. ${ }^{14}$ e aceito como referência nos ensaios biomecânicos de coluna lombar de suínos.

\section{Produção da fratura experimental}

Para realizar a fratura-explosão experimental foi utilizado o modelo descrito por Wang et al. ${ }^{15}$ que consiste na produção da falha óssea dos segmentos anterior e médio da coluna vertebral, conforme proposto por Denis como uma fratura-explosão ${ }^{5}$.

Após a aferição da altura (dimensão crânio-caudal) do corpo vertebral, foi determinado o corte em forma de cunha " $V$ " do $1 / 3$ médio do corpo vertebral que se estendia desde o ligamento longitudinal anterior até o ligamento longitudinal posterior. Este corte foi realizado de forma manual, com uma serra de fino calibre para que não houvesse lesão inadvertida dos outros segmentos (posterior). O bloco ósseo contendo o segmento anterior e médio da coluna vertebral foi destacado, simulando, portanto uma fraturaexplosão. Este modelo foi utilizado conforme preconizado em outros estudos biomecânicos já realizados ${ }^{15}$.

\section{Método de fixação pedicular}

Após o preparo das amostras e produção do modelo de fraturaexplosão, foi realizado a fixação pedicular no Grupo 3.

O local para a inserção do Pino de Schanz (NeoOrtho Produtos Ortopédicos SA, Curitiba, PR, BRA) foi determinado de acordo com a técnica de Magerl descrita na literatura médica ${ }^{16}$. Foi realizada a inserção manual de um guia inicial dentro do pedículo da vértebra para que fosse avaliada a penetração do Pino de Schanz. Após 
realizar a brocagem do pedículo, o Pino de Schanz foi inserido manualmente até o local determinado pelo guia. Os Pinos de Schanz utilizados apresentavam 5 milímetros $(\mathrm{mm})$ de diâmetro e $100 \mathrm{~mm}$ de comprimento.

Após inserção dos pinos, foram acoplados a rótula e as barras longitudinais do sistema de Pino de Schanz. Todo o sistema era apertado e calibrado com o instrumental desenvolvido para este sistema (Figura 1).
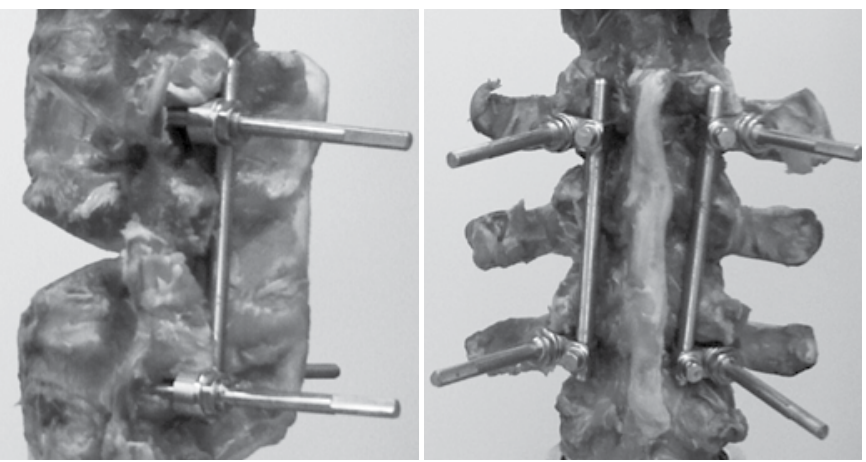

Figura 1. Reprodução da fratura-explosão e Fixação Pedicular Curta - Perfil (à esquerda) e Vista Posterior (à direita).

\section{Teste biomecânico}

Para realizar a fixação da amostra na máquina de testes servohidráulico, modelo Instron 3382 (Instron Co., Norwood, MA, EUA), foi criado um dispositivo de acoplamento. Este dispositivo consiste em um suporte de metal em forma de copo com profundidade central de $15 \mathrm{~mm}$ sobre o qual a amostra foi encaixada e fixada. Este dispositivo era acoplado na máquina de testes por uma rosca de metal na região superior do suporte.

A vértebra intermediária do segmento, $L 1$, foi centralizada no eixo e posicionada para se manter no plano horizontal após o acoplamento do dispositivo na máquina. O eixo de carga foi centralizado sobre o ligamento longitudinal posterior (LLP), local considerado como eixo axial do corpo vertebral ${ }^{17}$

Após encaixe de todo o conjunto (dispositivo de acoplamento e amostra), a máquina de testes era ligada e os parâmetros de força em Newtons $(\mathrm{N})$ e deslocamento em milímetros $(\mathrm{mm})$ eram zerados para calibragem do sistema (Figura 2).

O teste então era iniciado com uma taxa de deslocamento controlado de $5 \mathrm{~mm} / \mathrm{min}$. Os parâmetros de força e deslocamento eram automaticamente analisados pela máquina e os dados eram convertidos em um gráfico instantâneo. Estes parâmetros foram utilizados conforme estudo prévio de Lundin et al. ${ }^{14}$

Foi definido como falência (falha) da amostra, quando a curva do gráfico $(\mathrm{N} / \mathrm{mm})$ apresentava uma queda súbita durante o teste. $\mathrm{O}$ teste então era interrompido e o gráfico analisado preliminarmente.
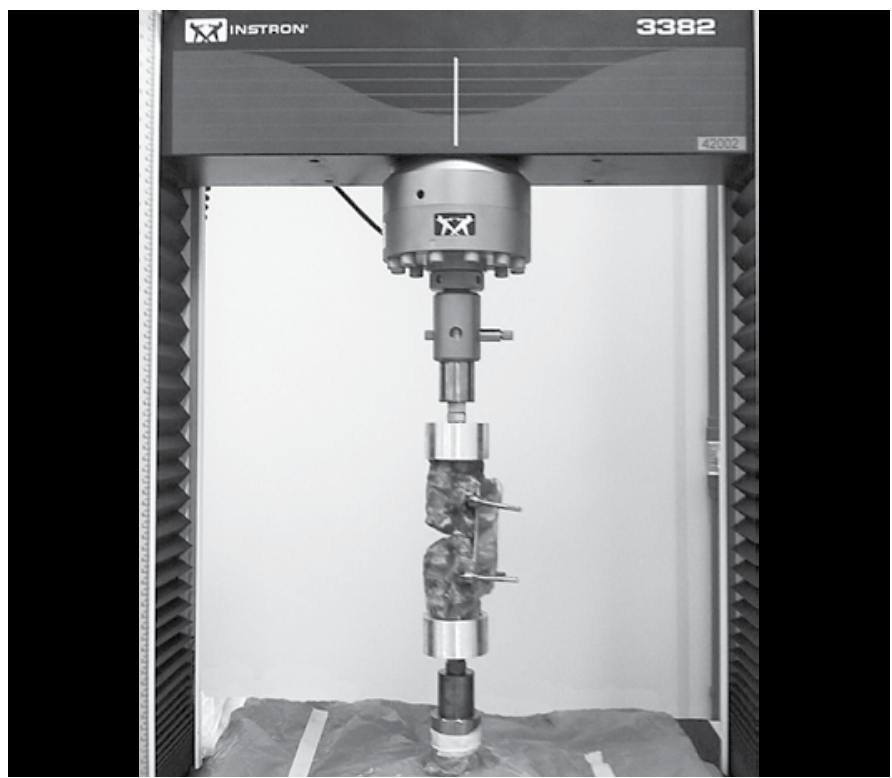

Figura 2. Amostra acoplada à máquina de testes.

\section{ANÁLISE DOS RESULTADOS}

Após a coleta dos dados do gráfico de força $(\mathrm{N})$ versus deslocamento $(\mathrm{mm})$, foi gerada uma tabela com o resultado de cada grupo de testes (Grupo 1, 2 e 3). Esta tabela continha os valores mensurados: Carga (N), Deslocamento $(\mathrm{mm})$ e a Rigidez $(\mathrm{N} / \mathrm{mm})$ na qual havia ocorrido a falência (falha) de cada amostra durante o teste biomecânico.

Estes dados foram então submetidos à análise estatística para validação das diferenças dos grupos do estudo. Foi aplicado o teste de sobrevivência de Kaplan-Meier para a verificação da diferença entre as amostras. As diferenças consideradas como estatisticamente significativas com o valor de $\mathrm{p}<0,05$ (IC-95\%) dados pelo teste de Logrank.

\section{RESULTADOS}

A Tabela 1 demonstra os resultados dos valores de Carga $(N)$, Deslocamento $(\mathrm{mm})$ e Rigidez $(\mathrm{N} / \mathrm{mm})$ dos grupos 1, 2 e 3 nos testes de compressão axial controlada

Quando comparamos os Grupos 1 e 2, verificamos que a rigidez das vértebras fraturadas (Grupo 2) apresentou somente $47 \%$ da rigidez das vértebras intactas (Grupo 1) durante o teste em compressão axial. Esta diferença foi estatisticamente significativa $(p<0,05)$

A comparação entre os Grupos 2 e 3 (vértebras fraturadas versus fixação pedicular curta), foi observado que o Grupo 3 apresentou

Tabela 1. Resultados dos testes mecânicos.

\begin{tabular}{|c|c|c|c|c|c|c|c|c|c|}
\hline \multirow[t]{2}{*}{ N. } & \multicolumn{3}{|c|}{ GRUPO 1-Vértebras Intactas } & \multicolumn{3}{|c|}{ GRUPO 2 -Vértebras fraturadas } & \multicolumn{3}{|c|}{ GRUPO 3 - Fixação Pedicular Curta } \\
\hline & Carga (N) & Deslocam (mm) & Rigidez (N/mm) & Carga (N) & Deslocam (mm) & Rigidez (N/mm) & Carga (N) & Deslocam (mm) & Rigidez (N/mm) \\
\hline 1 & 5351,09 & 14,35 & 372,69 & 1294,58 & 11,05 & 117,15 & 2116,39 & 10,23 & 206,82 \\
\hline 2 & 6254,61 & 19,10 & 327,33 & 2207,30 & 15,94 & 138,45 & 1835,91 & 8,25 & 222,32 \\
\hline 4 & 6251,98 & 14,60 & 427,98 & 2242,49 & 11,51 & 194,71 & 2131,62 & 11,08 & 192,33 \\
\hline 5 & 6726,62 & 14,56 & 461,77 & 3142,67 & 14,21 & 221,05 & 4112,60 & 10,10 & 407,18 \\
\hline 6 & 6548,89 & 15,27 & 428,84 & 2351,36 & 10,61 & 221,47 & 3302,69 & 10,44 & 316,28 \\
\hline 8 & 6674,84 & 15,63 & 426,83 & 3245,69 & 13,99 & 231,86 & 4264,80 & 9,05 & 471,24 \\
\hline 9 & 5874,98 & 14,74 & 398,43 & 4018,56 & 17,10 & 235,00 & 3023,83 & 8,53 & 354,37 \\
\hline 10 & 7124,65 & 17,76 & 400,96 & 1879,31 & 7,35 & 255,68 & 3342,51 & 10,21 & 327,37 \\
\hline Media & 6363,79 & 15,19 & 424,61 & 2591,39 & 12,93 & 200,50 & 2968,84 & 10,05 & 301,24 \\
\hline SD & 508,68 & 2,00 & 62,01 & 896,64 & 3,15 & 45,56 & 818,19 & 1,59 & 94,78 \\
\hline
\end{tabular}


uma rigidez 50\% maior do que o Grupo 2 durante o teste em compressão axial. Este aumento de $200,507 \mathrm{~N} / \mathrm{mm}$ para $301,243 \mathrm{~N} / \mathrm{mm}$ foi estatisticamente significativo $(p<0.05)$.

Ao efetuarmos as análises comparativas entre os Grupos 1 (vértebra intacta) e o Grupo 3 (fixação pedicular curta), verificamos que a rigidez do fixação pedicular curta foi $70 \%$ da rigidez das vértebras intactas. Estes dados indicam que a fixação pedicular curta não restaurou os valores de rigidez aos equivalentes de uma vértebra intacta. Estas diferenças foram estatisticamente significativas $(p<0.05)$ (Figura 3).

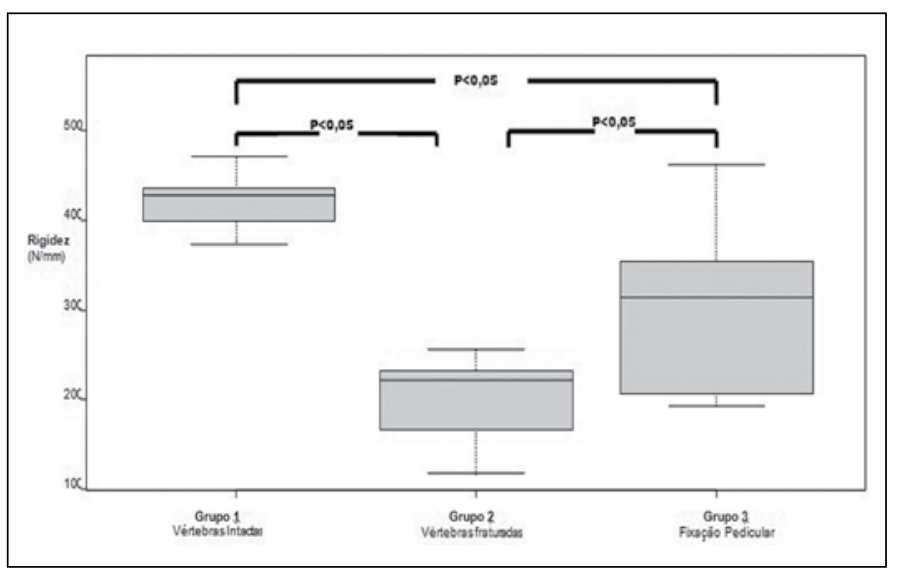

Figura 3 - Resultado da Rigidez Média e o Desvio Padrão para cada grupo. Diferenças estatísticas entre os grupos $(p<0,05)$.

\section{DISCUSSÃO}

A vértebra que apresenta uma fratura-explosão não transfere carga tão efetivamente quanto uma vértebra intacta. Esta análise biomecânica realizada demonstra que a fixação pedicular curta não fornece rigidez suficiente para promover estabilidade da fraturaexplosão quando submetida em carga axial de compressão. Este pode ser um dos motivos da ocorrência de falência da instrumentação ou perda da redução das fraturas durante a fase inicial do tratamento da fratura-explosão. O nosso estudo não utilizou parâmetros de amplitude de movimento nos três planos de movimento (flexão/extensão, lateralização direita/esquerda e rotação axial) porque a rigidez em compressão da estrutura testada reflete a capacidade de absorção de carga entre o implante a as colunas fraturadas. Por este motivo que somente a rigidez em compressão foi avaliada neste estudo. Os resultados dos testes em compressão axial foram semelhantes aos encontrados na literatura ${ }^{15,18}$

Não foram encontrados falhas ou quebras do material de instrumentação utilizado. Todos os instrumentais (pinos de Schanz, rótula conectora e barra longitudinal) foram retirados após cada teste e a integridade do material foi analisada. Não houve falha, quebra ou deformidade plástica em nenhum dos instrumentais utilizados e nas conexões entre eles (barra-rótula, pino-rótula), demonstrando que o instrumental utilizado não foi responsável pela diferença de rigidez entre os grupos. Esta análise nos permite considerar que a diferença de rigidez entre a coluna intacta (Grupo 1) e a coluna fraturada com fixação pedicular curta (Grupo 3), pode ser ocasionada pela falta de suporte anterior e médio da coluna vertebral e/ou a relação entre o tamanho da área de contato da interface pino-osso.

Uma das hipóteses aventadas após este estudo seria a avaliação da área de interface pino-osso. Considerando-se que esta área de contato fosse maior, a transferência da carga seria maior distribuída para o implante, consequentemente diminuindo a carga sobre o segmento fraturado. Este deve ser um dos motivos que a fixação pedicular longa tem se demonstrado superior a fixação pedicular curta em termos de rigidez biomecânica ${ }^{10,19}$. A fixação pedicular longa envolve múltiplos níveis, dividindo, portanto a carga entre todos os segmentos instrumentados, consequentemente diminuindo a carga individual na interface pino-osso de cada segmento. Uma das alternativas à fixação pedicular longa e que aumentaria a área de interface pino-osso, seria a fixação pedicular curta adicionando-se a fixação ao nível da fratura (fixação segmentar ou intermediária). O estudo publicado por Hart et al. ${ }^{20}$ demonstra que a fixação segmentar proporciona um aumento da rigidez do conjunto, porém este estudo foi realizado em segmentos íntegros de coluna vertebral, não foi realizado a análise deste tipo de fixação segmentar em condições que simulassem a fratura-explosão toracolombar. Mais estudos são necessários para avaliar a rigidez biomecânica de a fixação pedicular curta segmentar em situações que simulem a fratura-explosão toracolombar.

Utilizamos o modelo de produção de fratura-explosão que consiste na ressecção do segmento anterior e médio da coluna toracolombar, simulando o conceito de Denis em que a lesão do segmento anterior e médio proporciona instabilidade da coluna vertebral. Este modelo de ressecção em cunha de 1/3 do corpo vertebral, utilizados em estudos prévios, demonstrou a reprodutibilidade da fratura-explosão com instabilidade ${ }^{15}$. Os nossos resultados demonstraram que a coluna com fratura-explosão apresentou rigidez inferior a $50 \%$ da rigidez da coluna intacta, portanto, consideramos que este modelo de fratura ocasionou instabilidade suficiente para análise biomecânica. Não foi objetivo do nosso estudo de criar um novo modelo de reprodução de fratura-explosão toracolombar em suínos.

A coluna toracolombar do suíno adulto apresenta características morfológicas e biomecânicas semelhantes ao do ser humano mesmo sendo um animal quadrúpede ${ }^{21,22}$. Quando comparado com outros espécimes de animais (coelho, ovelha e porco jovem) reproduz com mais fidelidade o padrão de fratura toracolombar ${ }^{23}$. Optamos pela utilização de amostras de suíno, devido às dificuldades técnicas de obtenção de espécimes humanos, respeitando a legislação vigente e suas restrições adicionais (Lei 9.434, de fevereiro de 1997). Visando homogeneidade das amostras, todas foram pré-selecionadas de acordo com o peso e dimensões semelhantes. As amostras heterogêneas foram descartadas. A divisão das amostras foi realizada de forma aleatória entre os grupos distintos.

Algumas limitações do nosso estudo são observadas. A primeira diz respeito ao tipo teste biomecânico realizado. A coluna vertebral está sujeita a cargas em diversos planos: horizontal, vertical e rotacional. Haher et al. ${ }^{24}$ demonstraram que a ressecção da coluna anterior e média da coluna vertebral reduz a capacidade de transferência de carga em $70 \%$ quando submetidos a uma carga em compressão-flexão quando comparados com a coluna intacta. Isto demonstra que a rigidez biomecânica da coluna vertebral é menor em compressão-flexão quando comparado a testes em compressão axial pura. Utilizamos em nosso estudo o teste de compressão axial pura, portanto, para uma compreensão maior da falência da fixação pedicular curta, pode ser necessário a realização de testes biomecânicos com cargas compressivas associadas à flexão. Outra limitação do nosso estudo diz respeito ao modelo de fratura-explosão realizado. Algumas fraturas do tipo explosão ocasionam lesão da coluna posterior (laminas, facetas, pedículos vertebrais e ligamentos posteriores) além da lesão da coluna anterior e média. A reprodução este tipo de lesão complexa seria ideal para a validação das teorias do mecanismo da lesão, porém não seria consistente a reprodução seriada em testes experimentais, resultando em uma variabilidade acentuada de lesões. Portanto, para criar um padrão consistente de lesão, foi realizado no nosso estudo, um corte ósseo da coluna anterior e média de forma padronizada, permitindo simular a instabilidade neste tipo de fratura com o mínimo de erro metodológico. Estudos que reproduzissem a fratura-explosão das três colunas (anterior, média e posterior) de forma padronizada são necessários para avaliar o comportamento biomecânico da fixação pedicular curta. Outra limitação do nosso estudo envolve a utilização de coluna toracolombar de suínos e não 
de humanos. Existe diferença anatômica e mecânica entre a coluna de suíno e a de humanos, porém vários estudos foram publicados demonstrando que para testes biomecânicos, a utilização deste tipo de coluna é apropriada ${ }^{25}$. A quarta limitação é que o ambiente in vivo é parcialmente reproduzido pelos estudos in vitro, como realizado neste estudo.

\section{CONCLUSÃO}

A fixação pedicular curta não proporciona rigidez suficiente para restaurar a estabilidade equivalente a vértebra intacta durante os testes biomecânicos de compressão axial pura in vitro em modelos de fratura-explosão de suínos.

\section{REFERÊNCIAS}

1. Hu R, Mustard CA, Burns C. Epidemiology of incident spinal fracture in a complete population. Spine (Phila Pa 1976). 1996;21(4):492-9.

2. Esses SI, Botsford DJ, Kostuik JP. Evaluation of surgical treatment for burst fractures. Spine (Phila Pa 1976). 1990;15(7):667-73.

3. Holdsworth FW, Hardy A. Early treatment of paraplegia from fractures of the thoracolumbar spine. J Bone Joint Surg Br. 1953;35(4):540-50.

4. Holdsworth F. Fractures, dislocations, and fracture-dislocations of the spine. J Bone Joint Surg Am. 1970;52(8):1534-51.

5. Denis F. The three column spine and its significance in the classification of acute thoracolumbar spinal injuries. Spine (Phila Pa 1976). 1983;8(8):817-31.

6. Gertzbein SD. Scoliosis Research Society. Multicenter spine fracture study.Spine (Phila Pa 1976). 1992;17(5):528-40.

7. Korovessis PG, Baikousis A, Stamatakis M. Use of the Texas Scottish Rite Hospital instrumentation in the treatment of thoracolumbar injuries. Spine (Phila Pa 1976). 1997;22(8):882-8.

8. Shen WJ, Liu TJ, Shen YS. Nonoperative treatment versus posterior fixation for thoracolumbar junction burst fractures without neurologic deficit. Spine (Phila Pa 1976). 2001;26(9):1038-45

9. Akalm S, Ki M, Benli IT, Citak M, Mumcu EF, Tüzüner M. Results of the AO spinal internal fixator in the surgical treatment of thoracolumbar burst fractures. Eur Spine J. 1994:3(2):102-6.

10. McLain RF. The biomechanics of long versus short fixation for thoracolumbar spine fractures. Spine (Phila Pa 1976). 2006 May 15;31(11 Suppl):S70-9

11. Verlaan JJ, Diekerhof $\mathrm{CH}$, Buskens E, van der Tweel I, Verbout AJ, Dhert WJ et al. Surgical treatment of traumatic fractures of the thoracic and lumbar spine: a systematic review of the literature on techniques, complications, and outcome. Spine (Phila Pa 1976). 2004;29(7):803-14.

12. Jagodzinski $M$, Krettek $C$. Effect of mechanical stability on fracture healing-an update. Injury. 2007;38 (Suppl 1):S3-10.

13. Wilke HJ, Wenger K, Claes L. Testing criteria for spinal implants: recommendations for the standardization of in vitro stability testing of spinal implants. Eur Spine J. 1998;7(2):148-54

14. Lundin O, Ekström L, Hellström M, Holm S, Swärd L. Injuries in the adolescent porcine spine exposed to mechanical compression. Spine (Phila Pa 1976). 1998;23(23):2574-9.
15. Wang XY, Dai $L Y, X u H Z$, Chi YL. Biomechanical effect of the extent of vertebral body fracture on the thoracolumbar spine with pedicle screw fixation:an in vitro study. J Clin Neurosci. 2008:15(3):286-90.

16. Magerl FP. Stabilization of the lower thoracic and lumbar spine with external skeletal fixation. Clin Orthop Relat Res. 1984;(189):125-41.

17. Baranto A, Ekström L, Holm S, Hellström M, Hansson HA, Swärd L. Vertebral fractures and separations of endplates after traumatic loading of adolescent porcine spines with experimentally-induced disc degeneration. Clin Biomech (Bristol, Avon). 2005;20(10):1046-54.

18. Van Dieën JH, van der Veen A, van Royen BJ, Kingma I. Fatigue failure in shear loading of porcine lumbar spine segments. Spine (Phila Pa 1976). 2006;31(15):E494-8.

19. An HS, Singh K, Vaccaro AR, Wang G, Yoshida H, Eck J, et al. Biomechanical evaluation of contemporary posterior spinal internal fixation configurations in an unstable burstfracture calf spine model: special references of hook configurations and pedicle screws. Spine (Phila Pa 1976). 2004;29(3):257-62.

20. Hart R, Hettwer W, Liu Q, Prem S. Mechanical stiffness of segmental versus nonsegmental pedicle screw constructs: the effect of cross-links. Spine (Phila Pa 1976). 2006;31(2):E35-8.

21. Keller TS, Hansson TH, Holm SH, Pope MM, Spengler DM. In vivo creep behavior of the normal and degenerated porcine intervertebral disk: a preliminary report. J Spinal Disord. 1988;1(4):267-78.

22. SmitTH. The use of a quadruped as an in vivo model for the study of the spine - biomechanical considerations. Eur Spine J. 2002;11(2):137-44

23. Kettler A, Liakos L, Haegele B, Wilke HJ. Are the spines of calf, pig and sheep suitable models for pre-clinical implant tests? Eur Spine J. 2007:16(12):2186-92.

24. Haher TR, Tozzi JM, Lospinuso MF, Devlin V, O'Brien M, Tenant R, et al. The contribution of the three columns of the spine to spinal stability: a biomechanical model. Paraplegia. 1989;27(6):432-9.

25. Busscher I, van der Veen AJ, van Dieën JH, Kingma I Verkerke GJ, Veldhuizen AG. In vitro biomechanical characteristics of the spine: a comparison between human and porcine spinal segments. Spine (Phila Pa 1976). 2010;35(2):E35-42. 